\title{
PROKLA-Redaktion
}

\section{Editorial: Politische Ökonomie des Krieges}

$\mathrm{K}_{\mathrm{n}}^{\mathrm{r}}$

rieg und Rüstung waren bereits mehrfach Schwerpunkte unserer Zeitschrift, zuletzt in PROKLA 162 (Nie wieder Krieg?). Derzeit sind diese Themen aus der öffentlichen Wahrnehmung weitgehend verschwunden und wurden durch die Bedrohungen des Corona-Virus in den Schatten gestellt. Doch Tod und unendliches Leid durch blutige Auseinandersetzungen haben ebenso wenig aufgehört wie die Bestrebungen von vielen Staaten, sich immer größere Waffenarsenale zuzulegen.

"Friedensdividende" - so lautete vor drei Jahrzehnten ein hoffnungsvoller Slogan. Mit dem Ende des Kalten Krieges würden auch das Wettrüsten der beiden großen Blöcke und die von ihnen geführten Stellvertreterkriege beendet. Ost und West hatten gigantische konventionelle, atomare, chemische und biologische Arsenale aufgebaut, die nunmehr überflüssig schienen. Statt der laufenden Aufrüstung könnte eine kooperative Bearbeitung der Menschheitsprobleme beginnen. Bekanntlich kam es anders. Einige Jahre lang sanken die weltweiten Militärausgaben, doch dann stiegen sie wieder an und liegen seitdem höher als in den späten 1980er-Jahren. Daran sind vor allem zwei Länder beteiligt, die sich noch dazu in handelspolitische Auseinandersetzungen ver- strickt haben: die USA und China. Der US-amerikanische Militärhaushalt ist seit langem der größte der Welt und der militärisch-industrielle Komplex ist in den USA fest verankert. Seine Geschichte reicht bis zum Ersten Weltkrieg zurück, wie Frank Jacob anhand der anarchistischen Imperialismuskritik am Beispiel von Emma Goldmann und Alexander Berkman zeigt. Die beiden Anarchist_innen wurden während des Weltkriegs aufgrund ihrer Kritik an der Militarisierung und des sich formierenden militärisch-industriellen Komplexes des Landes verwiesen. Ideengeschichtlich wurden Kriegsökonomien jedoch häufig nicht nur negativ beurteilt, sondern durchaus auch als Konjunkturmotor gesehen - eine Einschätzung, die von Ökonom_innen und Historiker_innen, wie Renata Allio in ihrem Beitrag nachzeichnet, jedoch vielfach infrage gestellt wurde. Inzwischen ist es die Volksrepublik China, die den zweitgrößten Militärhaushalt aufweist. Das Land hat seine Militarisierung qualitativ wie quantitativ stark vorangetrieben. Es sieht danach aus, dass hier parallel zum ökonomischen Potenzial Chinas eine neue militärische Weltmacht entsteht. Für die USA und China (ebenso wie für Saudi-Arabien und Russland auf den folgenden Plätzen) bedeutet das nicht nur eine 
gigantische Vergeudung von Ressourcen. Die militärische Stärke soll auch demonstrieren, dass die Interessen des jeweiligen nationalen Kapitals notfalls gewaltsam durchzusetzen wären selbst wenn die US-amerikanische Regierung zumindest in Bezug auf neue Kampfhandlungen mittlerweile zu einer gewissen Zurückhaltung neigt. Der Vietnam-Krieg ebenso wie alle darauffolgenden militärischen Interventionen haben sich als extrem kostspielige Fehlschläge erwiesen. Dennoch stellt sich weiterhin die Frage, inwieweit imperialistische Ambitionen alter und neuer Wirtschaftsmächte kriegerisch durchgesetzt werden könnten. Auch die wachsende Militarisierung der EU sowie die ihrer Außengrenzen ist in diesem Kontext daher kritisch zu untersuchen. Gleiches gilt für das NATOMitglied Türkei. Axel Gehring analysiert in seinem Beitrag die polit-ökonomische Bedeutung der türkischen Rüstungsindustrie und die Versuche, eine import-substituierende Industrialisierung voranzutreiben.

In Anbetracht der Umbrüche der 1990er-Jahre riefen Politikwissenschaftler_innen um die Jahrtausendwende eine Epoche von "neuen Kriegen" aus, die an die Stelle der traditionellen zwischenstaatlichen Auseinandersetzungen getreten seien. Es handele sich dabei um unkonventionelle Konflikte, in denen verschiedene Bürgerkriegsparteien danach trachten, sich gewaltsam zu behaupten. Doch weder ist diese Form der Kriege wirklich neu, noch bedeutet sie, wirtschaftliche und militärische Großmächte hätten nichts mit ihnen zu tun - im Gegenteil. Der klassische Fall dafür ist der bald ein
Jahrzehnt andauernde Syrienkrieg mit bisher rund einer halben Million Toten und unzähligen Flüchtlingen. Zentrale Akteure sind die syrische Regierung und die sie bekämpfenden Rebellengruppen, außerdem Verbündete der Regierung wie Russland und Iran, regionale Mächte wie die Türkei, Saudi-Arabien und die Golfemirate, die westlichen Mitglieder der AntiIS-Koalition wie die USA, Frankreich, Großbritannien und die Bundesrepublik, schließlich der IS und das kurdische Rojava. Sie beteiligen sich auf unterschiedliche Weise am Krieg: mit regulären Truppen, mit Milizen, mit finanzieller oder logistischer Unterstützung, mit Waffenlieferungen und mit militärischer oder geheimdienstlicher Expertise. Dieser neuen Unübersichtlichkeit widmet sich Jens Warburg in seinem Beitrag zu den geopolitischen Umbrüchen und den Determinanten deutscher militarisierter Außenpolitik. Dabei wird in einer Rückschau die geopolitische Positionierung Deutschlands untersucht und gezeigt, dass und in welchem Sinne die Bundeswehr zu einem außenpolitischen Instrument wurde. Aber wie steht es dabei um ihr Verhältnis zur Rüstungsindustrie und um deren Beziehungen zum Verteidigungsministerium? Dieser Frage geht Dorothea Schmidt nach und fragt, ob es in Deutschland einen militärischindustriellen Komplex gibt, wobei sie drei Bereiche untersucht: die Beschaffungspolitik der Bundeswehr, die politische Förderung oder Begrenzung von Rüstungsexporten und die Aktivitäten »nicht legitimierter Machtzentren«, etwa innerhalb von Geheimdiensten wie dem BND. 
Wirtschaftliche Interessen der mächtigen Länder betreffen von jeher den Zugang zu Rohstoffen. Entsprechende Kriege gibt es seit Jahrhunderten, sie haben allerdings gerade in neuerer Zeit dazu geführt, dass Länder mit reichen natürlichen Vorkommen an Erdöl, Diamanten, Lithium oder Kobalt häufig als solche gesehen werden, die unter einem "Ressourcenfluch" leiden. Handelt es sich um schwache Staaten, so werden sie zum Kampffeld widerstreitender wirtschaftlicher wie politischer Interessen und bringen eine Form der Kriegsökonomie hervor, bei der Warlords das Geschäft des Krieges über Jahre hinweg betreiben können, indem sie sich durch den Zugriff auf ebendiese Ressourcen finanzieren - etwa in Angola, Sierra Leone, Libyen, Kolumbien oder Afghanistan. Gleichwohl können, dies zeigt Andreas Grünewald in seinem Beitrag auf, eine hohe Biodiversität und die Ausweisung von Naturschutzgebieten einer Militarisierung ebenfalls Vorschub leisten. Damit werden koloniale Bilder einer schützenswerten, unberührten Natur reproduziert und die Marginalisierung der lokalen Bevölkerung legitimiert. Diese Entwicklung und die Rolle der Deutschen Bundesregierung wie auch von großen Umweltverbänden, etwa dem WWF, veranschaulicht er in seinem Einspruch am Beispiel zahlreicher afrikanischer Schutzgebiete.

Geht es bei allen genannten Themen um Ursachen für die Militarisierung von Staaten, so stellt diese selbst von jeher ein Geschäftsfeld für einschlägige Unternehmen dar. Die Produktion und der Handel mit Waffen aller Art haben sich unterschiedlich entwickelt. Die Bestände von Atomwaffen in Ländern wie den
USA und Russland wurden reduziert, gleichzeitig werden sie allerdings laufend modernisiert. Bei schweren und leichten Waffen gab es einen Rückgang nach dem Ende des Kalten Krieges, doch hat sich dieser Trend seither umgekehrt. Nach wie vor sind es Kleinwaffen, die den allergrößten Teil der Kriegstoten weltweit verursachen. Um das Jahr 2000 war das ein großes Thema, was auch die UNO zu einem entsprechenden Aktionsprogramm veranlasste. Darüber hinaus wurden in der Bundesrepublik die Vorschriften zur Genehmigung von derartigen Exporten verschärft - aber an den realen Exporten hat das hier wie anderswo so gut wie nichts verändert. Gleichzeitig sind neue Bedrohungen durch Drohnenkriege und Risiken von Cyberkrieg entstanden, bei denen privates Kapital (wie im Fall von Huawei) eine Schlüsselrolle einnimmt. Tobias Biehle analysiert in seinem Beitrag die Bedeutung von Drohnen als Dual-UseTechnologie für die EU. Er analysiert anhand von Strategiepapieren die enge Verbindung von ziviler und militärischer Nutzung von Drohnen.

Außerhalb des Schwerpunkts wird dieses Heft durch zwei Beiträge ergänzt. Stefan Kerber-Clasen und Franziska MeyerLantzberg beleuchten kritisch die Debatte um Care. Sie argumentieren, dass die wachsende akademische und politische Aufmerksamkeit für die soziale Reproduktion einerseits einen Erfolg darstellt, andererseits jedoch eine stärkere Differenzierung des Care-Begriffs und der dort geleisteten Arbeit vorgenommen werden muss. Damit greifen sie unter anderem den Schwerpunkt der PROKLA 197 (Krise der Reproduktion) und den Beitrag »Das Gespenst der Care-Krise. 
Ein kritischer Blick auf eine aktuelle Debatte« von Friederike Maier und Dorothea Schmidt in PROKLA 195 auf. Christopher Wimmer beschäftigt sich in seinem Beitrag mit geschichtsphilosophischen Prämissen, die mitunter bei Marx zu finden sind und argumentiert, dass man mit Marx gegen Marx argumentieren kann, und sich Marx selbst zunehmend einem kontingenten Geschichtsverständnis angenähert habe. Dabei kommt, so Wimmer, dem Begriff des historischen Milieus eine zentrale Bedeutung zu.

\section{Der PROKLA Förderverein}

Die PROKLA erscheint seit 1971 und bietet politisch engagierte sozialwissenschaftliche und ökonomische Analysen. Allein von den Verkaufserlösen kann sich die PROKLA nicht finanzieren, und in die Abhängigkeit von Parteien oder großen Verlagen wollte sie sich nie begeben. Deshalb wird die PROKLA von einem Förderverein herausgegeben, der »Vereinigung zur Kritik der politischen Ökonomie e.V.«, die jährlich in ihrer Vollversammlung die Redaktion der Zeitschrift wählt und die nächsten Themenschwerpunkte diskutiert.

Kritische Sozialwissenschaft kann nicht dem Markt überlassen werden. Ohne solidarische Strukturen und finanzielle Unterstützung sind Zeitschriften wie die PROKLA kaum möglich. Die finanziellen Beiträge der Vereinsmitglieder ermöglichen das Erscheinen der PROKLA, sie schaffen die Voraussetzungen für Kontinuität und Planbarkeit, wie sie für die Redaktionsarbeit unabdingbar sind. Wir freuen uns über weitere Mitglieder, regelmäßige Spenden oder einmalige Zuwendungen. Weitere Informationen teilen wir gerne per E-Mail mit (redaktion@prokla.de).

Vereinigung zur Kritik der politischen Ökonomie e.V. | Postbank Berlin | IBAN: DE17 100100100538135100 | BIC: PBNKDEFF 